\title{
Towards a game-theoretic approach to content determination
}

\author{
Ralf Klabunde \\ Ruhr-Universität Bochum \\ Bochum, Germany \\ klabunde@linguistics.rub.de
}

\begin{abstract}
This paper argues for a game-theoretic approach to content determination that uses text-type specific strategies in order to determine the optimal content for various user types. By means of content determination for the description of numerical data the benefits of a game-theoretic treatment of content determination are outlined.
\end{abstract}

\section{Introduction}

This is a programmatic paper on the principles of content determination in natural language generation (NLG). It arose from the insight that we do not know much about the underlying principles and computational properties of several tasks in NLG. Especially conceptualization - the selection of the information to be conveyed in a natural language text, and the adaptation of this information to the language-specific requirements - is still a white spot on the generation map (Guhe, 2007). Content determination is that sub-process during conceptualization that is responsible for the selection of the information to be conveyed and its ordering. Several authors assume that establishing rhetorical relations between informational units and the successive construction of tree structures for the overall information should also be considered as a content determination task (see, e.g. Reiter and Dale (2000)), but I will ignore this task in this paper and confine my considerations to the selection and ordering of informational units, in particular propositions.

Content determination is coupled with the linguistic domain in two ways, since the content does not only need to be expressible in the target language, but the determination process is already affected by pragmatic organisation principles for specific text types. I am convinced that game the- ory is the appropriate tool for a formulation of these principles.

In what follows, I will first explain why content determination should be viewed as a game played by the speaker/system $\mathcal{S}$ and the speaker's/system's representation of a listener/user $\mathcal{L}$ - the 'user model'. After that I will outline the different strategies relevant for content determination by means of the content for usertailored descriptions of numerical data.

\section{Appproaches to content determination in NLG}

The various approaches to content determination proposed in the literature may be classified in a two-dimensional way, viz. with respect to information flow (top down vs. bottom-up), and with respect to the methods used (reasoning or the use of schemas).

From an engineering perspective - the dominant view in NLG - a top-down approach, focusing on the communicative goal and using schemas which determine where to realize which information, is the most attractive and most often method used, although it lacks of a theoretical grounding. A deep reasoning approach would thus be more attractive, but is not always feasible in practice.

One of the problems in content determination is that the amount and quality of the information to be conveyed depends on the interests and cognitive abilities of the respective user and the coherence requirement. Content determination is selecting material from the domain in the hope that it will permit a coherent realization as a text. Hence, this sub-task should be viewed as a process that is also constrained by pragmatic principles for establishing coherence.

I proceed on the assumption that a theoretically well-founded reasoning approach can be established within the framework of analytic game theory (see, e.g., Shoham and Leyton-Brown (2009)). 
The benefit of a game theoretic treatment is its focus on interacting agents and the reasoning mechanisms associated with games: If we are able to show that the content to be conveyed is determined by concepts of rational interaction, then we get insights into the principles that guide the overall content determination process.

The basic ideas are as follows: First, the random device - used in game-theoretic pragmatics to provide $\mathcal{S}$ with some meaning - must be replaced by a function that maps informational units of the domain to propositions. Additionally, $\mathcal{L}$ 's reasoning capabilities are taken into account. The interplay of both components reflects $\mathcal{S}$ 's cognitive effort to construct the proposition and represents some of the adaptive cognitive mechanisms of $\mathcal{S}$. It is well known from pragmatic and psycholinguistic studies that speakers do not only try to minimize their own effort in the production process, but that they take into account features of the listener and adopt content and form of their utterance to the listener's assumed cognitive and linguistic capabilities. Hence, the process of content determination is guided by speaker-strategies and adaptation processes which should be modelled as adopted addressee-strategies. Under this view, the ultimate goal of content determination is to find a coherent catenation of propositions that is tailored to the addressee: $\mathcal{S}$ is a decision-maker and she is already playing with $\mathcal{L}$ at pre-linguistic stages.

\section{Game theoretic pragmatics}

Jäger (2007) describes the conception of gametheoretic pragmatic analyses as follows: A game is an utterance situation with a speaker $\mathcal{S}$ and a hearer $\mathcal{L}$ as players. The actions performed by these players are the production and interpretation of utterances, and the payoffs represent the cognitive and linguistic expenses of both players. If a set $M$ of meanings is given and a set $F$ of linguistic forms, a speaker strategy $s$ is a function from $M$ to $F$. Accordingly, a hearer strategy $h$ is a function from $F$ to $M$. In this paper, I assume that $M$ is a set of propositions, i.e. a set of situative, truth-functional, concepts.

Within this framework, the production process is treated in a simplifying way. A random device assigns some meaning $m \in M$ to $\mathcal{S}$ who has to select an appropriate form $f \in F$. Successful communication is given if $\mathcal{L}$ is able to reconstruct $m$ from $f$. The $\delta$-function defines just this:

$$
\delta_{m}(s, h)= \begin{cases}1 & \text { iff } h(s(m))=m \\ 0 & \text { otherwise }\end{cases}
$$

$\mathcal{S}$ has a choice between simple or more complex expressions to express the meaning $m$. In order to measure this complexity, a function cost from $F$ to the nonnegative real numbers is given whose exact shape is of no interest for this paper. The speaker utility $u_{s}$ refers to the cost-function in addition to some positive coefficient $k$ that represents the speaker's priorities. A low value of $k$ indicates that communicative success is more important than minimal effort, and a high value of $k$ means that effort is more important than success.

$$
u_{s}(m, s, h)=\delta_{m}(s, h)-k \times \operatorname{cost}(s(m))
$$

The addressee's utility can be identified with the $\delta$-function:

$$
u_{h}(m, s, h)=\delta_{m}(s, h)
$$

In order to adopt Jäger's characterization of a game-theoretic model of communication to NLG purposes, one has to modify it into two directions. The minor change concerns the random device that assigns meanings to the speaker. I replace this device by a function $i$ that maps informational units $d$ of the domain $D$ to propositions $p \in M$. The production grammar $s$ picks up these propositions and transforms them into linguistic forms $f$.

The more substantial change concerns the hearer strategy. From an NLG perspective, one is not primarily interested in a hearer strategy that maps forms to meanings, but in the effect of the conveyed information w.r.t. the hearer's information state $T_{\mathcal{L}}$. The aim of $\mathcal{S}$ is to modify $T_{\mathcal{L}}$, but it is $\mathcal{L}$ who decides how to process the information conveyed by $\mathcal{S}$. In general, $\mathcal{L}$ 's interpretation task is to find an explanation for $p$ on the basis of his own beliefs. In other words, interpretation is abductive reasoning (Hobbs et al., 1993). Suppose $\mathcal{S}$ conveys a set of propositions $A$. Then the actions available to $\mathcal{L}$ - if $A$ is new information for him - are several update mechanisms $u p\left(T_{\mathcal{L}}, A\right)$. He may just add $A$ to $T_{\mathcal{L}}$ and accept $A$ as new information without finding any explanation for $A$ : $u p\left(T_{\mathcal{L}}, A\right)=\left(T_{\mathcal{L}} \cup A\right) \neq T_{\mathcal{L}}$. The other extreme would be to compute the set of all logical conse- 
quences of $T_{\mathcal{L}} \cup A$, i.e. $u p\left(T_{\mathcal{L}}, A\right)=C n\left(T_{\mathcal{L}} \cup A\right) .{ }^{1}$ However, this set is just the ideal state of a logically omniscient person; a more realistic view is to characterize the strategies of $\mathcal{L}$ by different depths in reasoning, starting from depth $=0$ (i.e. $T_{\mathcal{L}} \cup A$ ) up to some information state close to $C n\left(T_{\mathcal{L}} \cup A\right)$. I use $u p\left(T_{\mathcal{L}}, A\right) \prec C n\left(T_{\mathcal{L}} \cup A\right)$ to represent this state. Note that $u p\left(T_{\mathcal{L}}, A\right) \prec C n\left(T_{\mathcal{L}} \cup A\right)$ is not a fixed information state but depends on the user type. If the players want to be communicatively successful, $\mathcal{L}$ should ideally try to find an explanation for $A$ that results in that mentioned information state. Hence, communicative success with respect to a single proposition $p$ may now be defined by:

$\delta_{d}(s, h, i, u p)= \begin{cases}1 & \text { iff } h(s(i(d)))=i(d)=p \\ \text { and } & \\ & u p\left(T_{\mathcal{L}}, p\right) \prec C n\left(T_{\mathcal{L}} \cup\{p\}\right) \\ 0 & \text { otherwise }\end{cases}$

The speaker utility is defined as:

$$
u_{s}(s, h, i, u p)=\delta_{d}(s, h, i, u p)-k \times \operatorname{cost}(i(d))
$$

and the hearer utility is

$$
u_{h}(s, h, i, u p)=\delta_{d}(s, h, i, u p)
$$

Within this overall picture of information exchange and communicative success, content determination is the interplay of $i$ with $u p\left(T_{\mathcal{L}}, \operatorname{ran}(i)\right)$, i.e. the update of $\mathcal{L}$ 's information state with the range of $i$. In the rest of this paper I will show by means of an example how this interplay can be spelled out in detail. As will hopefully become apparent, the approach amounts to some sort of game - a game that takes into account specific strategies of $\mathcal{S}$ and the abductive inference strategies of $\mathcal{L}$ to create a content that is optimal for both.

\section{Content determination for reports of numerical data}

Let us assume that the content underlying reports of performance data shall be tailored to an expert and a layman, respectively. The essential conceptualization process for content of this type is the summarization of numerical data to propositional units that are tailored to the addressee's needs. I

\footnotetext{
${ }^{1}$ Consider that abduction in its simplest form can be reformulated in deductive terms.
}

use normal form games for this task in which the expertises of the users are reflected in different Nash equilibria. $\mathcal{L}$ as expert requires content with a different equilibrium than $\mathcal{L}$ as layman does.

The basic scenario is as follows: A speedometer $f$ provides data about speed and the distance covered during a cycling tour. These numerical data shall be transformed into propositional units that are optimal for the respective user types. For reasons of clarity, let us assume two different user types only, a training expert and a layman who want to receive a detailed and a more shallow description, respectively. In both cases the actual values recorded by the speedometer will be compared with ideal training values, and the deviations from these ideal values shall be reported in the generated text.

Of course, the main task for $\mathcal{S}$ is to summarize these numerical data in single propositions, but how to determine the amount of data to be put into one proposition? I assume that $\mathcal{S}$ 's side of the coin is an approximation problem. The grade of detail required for the expert and the layman shall be given by an approximation $a$ of the function $f$. Let us assume that the approximation is $1 / 10$ for the expert and $1 / 5$ for the layman $(\forall x \in \operatorname{dom}(f)$ : $a(x)=x \pm x / 10$ or $a(x)=x \pm x / 5)$. Table 1 shows an exemplary function for the first seven measure points and the approximations used.

\begin{tabular}{lllll} 
distance & $\begin{array}{l}\text { speed } \\
\mathrm{n}\end{array}$ & $\begin{array}{l}\text { ideal } \\
\text { value }\end{array}$ & $\begin{array}{l}\text { approx. } \\
1 / 10\end{array}$ & $\begin{array}{l}\text { approx. } \\
1 / 5\end{array}$ \\
\hline 1 & 25.3 & 25 & $22.5-27.5$ & $20.0-30.0$ \\
2 & 28.2 & 26 & $23.4-28.6$ & $20.8-31.2$ \\
3 & 31.7 & 27 & $24.3-29.7$ & $21.6-32.4$ \\
4 & 30.5 & 28 & $25.2-30.8$ & $22.4-33.6$ \\
5 & 32.8 & 29 & $26.1-31.9$ & $23.2-34.8$ \\
6 & 31.1 & 30 & $27.0-33.0$ & $24.0-36.0$ \\
7 & 25.8 & 30 & $27.0-33.0$ & $24.0-36.0$ \\
$\vdots$ & $\vdots$ & $\vdots$ & $\vdots$ & $\vdots$
\end{tabular}

Table 1: Some numerical data

In addition to the values that are outside of the approximations, the degree of exceeding or going below the ideal value should be taken into account as well. We do not just want to generate a sentence like at kilometer 3 you went too fast if the actual values were outside the approximation hull and much higher than the ideal one, but at kilometer 3 you went much too fast. Therefore, we define a threshold such that every value above that threshold will be classified as being much higher than 
the ideal value, and all values below that threshold are classified as being an exiguous deviation from that ideal value. Then the six relevant speaker actions are $\mathrm{N}-0, \mathrm{~N}-1,1 / 10-0,1 / 10-1,1 / 5-0$ and 1/5-1 with 0 and 1 indicating no use of a threshold and the use of one relevant threshold, respectively.

According to section 3, the strategies of $\mathcal{L}$ concern the interpretation grammar, i.e. the mapping from linguistic forms to propositions $(h: F \rightarrow P)$ and an update of $\mathcal{L}$ 's information state that may include (abductive) reasoning. The abductive inferences drawn by the layman differ from those of the expert by the profundity of the explanation: While the layman is primarily interested in increasing his fitness, the expert should be interested in a more profound explanation. Let us assume three update strategies: NOINFERENCES, i.e. up $\left(T_{\mathcal{L}}, P\right)=T_{\mathcal{L}} \cup P$, EXHaustiveReasoning, i.e. $u p\left(T_{\mathcal{L}}, P\right)=\left(T_{\mathcal{L}} \cup P\right) \prec C n\left(T_{\mathcal{L}} \cup P\right)$, and MUNDANEREASONING, i.e. reasoning with only a very limited number of inferences involved.

The payoffs for the players may be motivated as follows. For $\mathcal{S}$ holds: A more detailed content requires more effort in providing that content. Furthermore, realizing the degree of exceeding the value requires additional cognitive effort. Since $\mathcal{S}$ pursues to reduce her efforts, the highest payoff will be associated with the lowest effort. The more detailed the content is, the lesser is $\mathcal{L}$ 's effort to reason. However, a text that explains everything violates the Gricean maxim of quantity. Therefore, $\mathcal{L}$ should prefer at least mundane reasoning, and we could motivate the listener's payoffs by the number of inferences to be drawn.

The utility matrix in Table 2 shows the action combinations of $\mathcal{S}$ and $\mathcal{L}$ as layman. The Nash equilibrium is the strategy $(1 / 5-0$, MUNDANEREASONING); $\mathcal{S}$ will generate propositions that comprise the numerical data outside of the widest approximation hull, and without any further differentiation w.r.t. the degree of exceeding the ideal values. $\mathcal{S}$ knows that $\mathcal{L}$ 's interpretation of the propositions is an abductive proof graph that represents a simple explanation of them.

With $\mathcal{L}$ as expert the payoffs must be swapped. Since the expert is able to find a more profound explanation, he strives for exhaustive reasoning. $\mathcal{S}$, in turn, knows this and will therefore select the smaller approximation. Hence, we get the utility matrix in Table 3 with (1/10-0, EXHAUSTIVEREASONING) as Nash equilibrium.

\begin{tabular}{llcc}
\hline & NOINF. & MUNDANER. & EXH.R. \\
N-0 & 1,5 & 1,7 & 1,1 \\
N-1 & 0,6 & 0,8 & 0,2 \\
$1 / 10-0$ & 3,5 & 3,7 & 3,1 \\
$1 / 10-1$ & 2,6 & 2,8 & 2,2 \\
$1 / 5-0$ & 6,5 & 6,7 & 6,1 \\
$1 / 5-1$ & 5,6 & 5,8 & 5,2 \\
\hline
\end{tabular}

Table 2: Utility matrix with $\mathcal{L}$ as layman

\begin{tabular}{llcc}
\hline & NOINF. & MUNDANER. & EXH.R. \\
$\mathrm{N}-0$ & 1,5 & 1,1 & 1,7 \\
$\mathrm{~N}-1$ & 0,6 & 0,2 & 0,8 \\
$1 / 10-0$ & 6,5 & 6,1 & 6,7 \\
$1 / 10-1$ & 5,6 & 5,2 & 5,8 \\
$1 / 5-0$ & 3,5 & 3,1 & 3,7 \\
$1 / 5-1$ & 2,6 & 2,2 & 2,8 \\
\hline
\end{tabular}

Table 3: Utility matrix with $\mathcal{L}$ as expert

\section{Outlook}

Due to the programmatic status of this paper, several issues have been omitted we will deal with in our future work. The most pressing tasks concern the action sets of $\mathcal{S}$ and $\mathcal{L}$ that must be refined, and the payoffs must be based on empirical observations. However, as sketchy as the given example may be, it shows that NLG stands to benefit from Game Theory.

\section{References}

Markus Guhe. 2007. Incremental Conceptualization for Language Production. Lawrence Erlbaum, Mahwah, NJ.

Jerry Hobbs, Mark Stickel, Douglas Appelt, and Paul Martin. 1993. Interpretation as Abduction. Artificial Intelligence, 63, 69-142.

Gerhard Jäger. 2007. Evolutionary Game Theory and typology: a case study. Language, 83, 74-109.

Ehud Reiter and Robert Dale. 2000. Building Natural Language Generation Systems. Cambridge University Press, Cambridge.

Yoav Shoham and Kevin Leyton-Brown. 2009. Multiagent Systems: Algorithmic, Game-theoretic and Logical Foundations. Cambridge University Press, Cambridge. 